TAIWANESE JOURNAL OF MATHEMATICS

Vol. 17, No. 1, pp. 333-350, February 2013

DOI: $10.11650 /$ tjm.17.2013.1728

This paper is available online at http://journal.taiwanmathsoc.org.tw

\title{
LINEAR MULTIFRACTIONAL STOCHASTIC VOLTERRA INTEGRO-DIFFERENTIAL EQUATIONS
}

\author{
Nguyen Tien Dung
}

\begin{abstract}
In this paper we prove the variation of parameters formula for linear Volterra integro-differential equations driven by multifractional Brownian motion. To do this, an approximation result for the Stratonovich stochastic integral with respect to the multifractional Brownian motion is given. Based on our obtained results we study the almost sure exponential convergence of the solution. Also, the existence and uniqueness of the solution of a multifractional Volterra integrodifferential equation with time delay are proved.
\end{abstract}

\section{INTRODUCTION}

Let $H:[0,+\infty) \rightarrow(0,1)$ be a Hölder function of exponent $\beta>0$, i.e. for any $t_{1}, t_{2} \in[0,+\infty)$ such that $\left|t_{1}-t_{2}\right|<1$, there exists a constant $c_{0}>0$ such that

$$
\left|H_{t_{1}}-H_{t_{2}}\right| \leq c_{0}\left|t_{1}-t_{2}\right|^{\beta} \text {. }
$$

According to the definition given by Peltier and Lévy-Véhel in [12], a multifractional Brownian motion $(\mathrm{mBm}), B_{t}^{H_{t}}$, with Hurst functional parameter $H_{t} \in(0,1)$ is a stochastic process of the following form

$$
B_{t}^{H_{t}}=\frac{1}{\Gamma\left(H_{t}+\frac{1}{2}\right)}\left(\int_{-\infty}^{0}\left[(t-s)^{H_{t}-\frac{1}{2}}-(-s)^{H_{t}-\frac{1}{2}}\right] d W_{s}+\int_{0}^{t}(t-s)^{H_{t}-\frac{1}{2}} d W_{s}\right)
$$

where $W_{s}$ is a standard Brownian motion. In the case, where $H_{t}$ is a constant, $\mathrm{mBm}$ reduces to fractional Brownian motion ( $\mathrm{fBm})$.

As showed in the literature (for example, see $[3,7,12]$ ), $\mathrm{mBm}$ seems to be a more flexible model than $\mathrm{fBm}$. The almost sure Hölder exponent of $\mathrm{mBm}$ is allowed to vary

Received February 29, 2012, accepted July 26, 2012.

Communicated by Yuh-Jia Lee.

2010 Mathematics Subject Classification: 45D05, 60G22, 60H07.

Key words and phrases: Volterra integro-differential equations, Variation of parameters formula, Multifractional Brownian motion, Malliavin calculus. 
along the trajectory, a useful feature when one needs to model processes whose regularity depends in time, such as Internet traffic or images. Because of these advantages, it is desirable to construct the stochastic integral with respect to $\mathrm{mBm}$, as well as to study the related problems. However, the same as $\mathrm{fBm}, \mathrm{mBm}$ is not a semimartingale if $H_{t} \neq \frac{1}{2}$, and we cannot use the classical Itô theory in our context.

Several authors considered the second term in the right hand side of (1.1) as a definition of $\mathrm{mBm}$ which is called a $\mathrm{mBm}$ of the Riemann-Liouville type (RL-mBm) (for example, see [8]). More precisely, we use the following definition for RL-mBm.

Definition 1.1. Let $H:[0,+\infty) \rightarrow(0,1)$ be a Hölder function of exponent $\beta>0$. The multifractional Brownian motion of the Riemann-Liouville form (RL$\mathrm{mBm}$ ) with Hurst functional parameter $H \in(0,1)$, denoted by $\left\{W_{t}^{H_{t}}, t \geq 0\right\}$, is a centered Gaussian process defined by

$$
W_{t}^{H_{t}}=\int_{0}^{t}(t-s)^{\alpha_{t}} d W_{s} \quad, \alpha_{t}=H_{t}-\frac{1}{2} .
$$

Throughout the paper, we assume that $H_{t}$ is a continuously differentiable function on $[0,+\infty)$. Since RL-mBm is a Gaussian process, we can use the stochastic calculus developed by Alòs et al. in [1] to get a definition for stochastic integral with respect to $W^{H_{t}}$, named the Stratonovich integral. In this paper we use Stratonovich integral with respect to RL-mBm with Hurst functional parameter $H_{t}>\frac{1}{2}$ to study linear multifractional stochastic Volterra integro-differential equations that are described in forms

$$
d X_{t}=\left(a X_{t}+\int_{0}^{t} G(t-u) X_{u} d u\right) d t+\sigma_{t} d W_{t}^{H_{t}},
$$

the initial condition $X_{0}=x$ is a real constant.

The volatility $\sigma_{t}$ does not depend on $X_{t}$, we can employ the traditional iteration method to prove the existence and uniqueness of the solution. After doing this, we develop a semimartingale approximate approach to prove a variation of parameters formula for the solution which plays an important role in theory of Volterra equations. Our obtained formula allows us to study exponential convergence of the solution via deterministic Volterra equations. Moreover, it also plays a key role in proving the existence and uniqueness of the solution for the stochastic delay Volterra equation driven by RL-mBm:

$$
d X_{t}=\left(a X_{t}+\int_{0}^{t} G(t-u) X_{u} d u\right) d t+b\left(X_{t-r}\right) d W_{t}^{H_{t}},
$$

$X_{t}=\phi(t), t \in[-r, 0]$, where $\phi \in C[-r, 0]$. 
This paper is organized as follows: In Section 2, we recall some fundamental facts about the Malliavin calculus and introduce the Stratonovich stochastic integral with respect to RL-mBm. We also point out that RL-mBm as well as stochastic integral with respect it can be approximated by semimartingales. Section 3 contains the main results of this paper regarding the equation (1.3) which proved the variation of parameters formula for the solution and the almost sure exponential convergence of the solution to zero. Section 4 is devoted to study the multifractional stochastic Volterra integro-differential equations with time delay.

\section{Stratonovich Integral with Respect to RL-mBm}

The aim of this section is to introduce the Stratonovich stochastic integral with respect to RL-mBm with Hurst parameter $H_{t}>1 / 2$ and being a continuously differentiable function.

First of all, let us recall some elements of stochastic calculus of variations with respect Brownian motion. Fixed $T>0$, for $h \in L^{2}([0, T], \mathbb{R})$, we denote by $W(h)$ the Wiener integral

$$
W(h)=\int_{0}^{T} h(t) d W_{t} .
$$

Let $\mathcal{S}$ denote the dense subset of $L^{2}(\Omega, \mathcal{F}, P)$ consisting of those classes of random variables of the form

$$
F=f\left(W\left(h_{1}\right), \ldots, W\left(h_{n}\right)\right),
$$

where $n \in \mathbb{N}, f \in C_{b}^{\infty}\left(\mathbb{R}^{n}, L^{2}([0, T], \mathbb{R})\right), h_{1}, \ldots, h_{n} \in L^{2}([0, T], \mathbb{R})$. If $F$ has the form (2.1), we define its derivative as the process $D^{W} F:=\left\{D_{t}^{W} F, t \in[0, T]\right\}$ given by

$$
D_{t}^{W} F=\sum_{k=1}^{n} \frac{\partial f}{\partial x_{k}}\left(W\left(h_{1}\right), \ldots, W\left(h_{n}\right)\right) h_{k}(t) .
$$

More generally, for each $k \geq 1$ we can define the iterated derivative operator on a cylindrical random variable by setting

$$
D_{t_{1}, \ldots, t_{k}}^{W, k} F=D_{t_{1}}^{W} \ldots D_{t_{k}}^{W} F
$$

For any $p \geq 1$ we shall denote by $\mathbb{D}_{W}^{1, p}$ the closure of $\mathcal{S}$ with respect to the norm

$$
\|F\|_{1, p}:=\left(E|F|^{p}+\int_{0}^{T} E\left|D_{r}^{W} F\right|^{p} d r\right)^{\frac{1}{p}} .
$$


We now apply the stochastic calculus with respect to Gaussian processes developed by Alòs et al. [1] to introduce a definition of stochastic integration with respect to $\mathrm{RL}-\mathrm{mBm} W_{t}^{H_{t}}$. In [1], for regular kernels, the following hypothesis is required.

Hypothesis (K4). For all $s \in[0, T), K(., s)$ has bounded variation on the interval $(s, T]$ and $\int_{0}^{T}|K|((s, T], s)^{2} d s<\infty$ (where, for all $s \in[0, T),|K|((s, T], s)$ denotes the total variation of $K(., s)$ on $(s, T])$.

In our context, it is obvious that the kernel $K(t, s)=(t-s)^{\alpha_{t}}$ fulfils the hypothesis (K4) because $\alpha_{t}=H_{t}-\frac{1}{2}$ is a differentiable function with bounded derivative in $[0, T]$.

In the remaining of the paper, we set $K(t, s):=(t-s)^{\alpha_{t}}$. Denote by $\mathcal{E}$ the set of step functions on $[0, T]$ and consider the norm on $\mathcal{E}$ :

$$
\|\varphi\|_{K}^{2}:=\int_{0}^{T}\left(\int_{s}^{T}\left|\varphi_{t}\right| \partial_{1} K(t, s) d t\right)^{2} d s
$$

where $\partial_{1} K(t, s)=\frac{\partial}{\partial t} K(t, s)$. The completion of $\mathcal{E}$ with respect to the $\|\cdot\|_{K}$-norm will be denoted by $\mathcal{H}_{K r}$. Also denote by $\mathbb{D}_{W}^{1,2}\left(\mathcal{H}_{K r}\right)$ the space of stochastic processes satisfying the following two conditions:

$$
E\|u\|_{K}^{2}=E \int_{0}^{T}\left(\int_{s}^{T}\left|u_{t}\right| \partial_{1} K(t, s) d t\right)^{2} d s<\infty
$$

and

$$
E \int_{0}^{T}\left\|D_{r}^{W} u\right\|_{K}^{2} d r=E \int_{0}^{T} \int_{0}^{T}\left(\int_{s}^{T}\left|D_{r}^{W} u_{t}\right| \partial_{1} K(t, s) d t\right)^{2} d s d r<\infty .
$$

Let $u=\left\{u_{t}, t \in[0, T]\right\}$ be a stochastic process in $\mathbb{D}_{W}^{1,2}\left(\mathcal{H}_{K r}\right)$, one can define the divergence integral of $u$ with repsect to RL-mBm by (see formula (21) in [1])

$$
\int_{0}^{t} u_{s} \delta W_{s}^{H_{s}}=\int_{0}^{t}\left(\int_{r}^{t} u_{s} \partial_{1} K(s, r) d s\right) \delta W_{r}
$$

where $\delta W_{r}$ is Skorohod differential. Moreover, $r \mapsto \int_{r}^{t} u_{s} \partial_{1} K(s, r) d s$ is Stratonovich integrable with respect to $W$. By taking into account the relation between Skorohod integral and Stratonovich integral, we use in this paper the following definition for mutifractional stochastic integral. 
Definition 2.1. Assume that $H_{t}>1 / 2$ and is a continuously differentiable function. Let $u \in \mathbb{D}_{W}^{1,2}\left(\mathcal{H}_{K r}\right)$, the Stratonovich stochastic integral of $u$ with respect to RL-mBm, $W^{H_{t}}$, is defined by

$$
\int_{0}^{t} u_{s} d W_{s}^{H_{s}}=\int_{0}^{t} u_{s} \delta W_{s}^{H_{s}}+\int_{0}^{t} \int_{s}^{t} D_{s}^{W} u_{r} \partial_{1} K(r, s) d r d s,
$$

where $\partial_{1} K(r, s)=(r-s)^{\alpha_{r}-1}\left[\alpha_{r}^{\prime}(r-s) \ln (r-s)+\alpha_{r}\right]$.

Remark 2.1. When $H_{t}=H$ is a constant, the divergence integral with respect to fBm with $H \in(0,1)$ has also been defined by Privault in [13, Definition 10].

Proposition 2.1. Let $u=\left\{u_{t}, 0 \leq t \leq T\right\}$ be an adapted process bounded in the norm of the space $\mathbb{D}_{W}^{1,2}$, i.e.

$$
\sup _{0 \leq t \leq T}\left(E\left|u_{t}\right|^{2}+\int_{0}^{T} E\left|D_{r}^{W} u_{t}\right|^{2} d r\right)<\infty .
$$

Then $u \in \mathbb{D}_{W}^{1,2}\left(\mathcal{H}_{K r}\right)$.

Proof. By using Hölder inequality it is easy to check the conditions (C1) and (C2).

We end this section by showing that RL-mBm can be approximated by semimartingales. This approximation will be the background of the paper and before stating it in Theorem 2.1, let us recall a fundamental property of RL-mBm.

Lemma 2.1. Let $\left\{W_{t}^{H_{t}}, 0 \leq t \leq T\right\}$ be a $R L-m B m$ with $H_{t} \in(0,1)$. Then for each $p>0$, there exists $C_{T, p}>0$ such that

$$
E\left|W_{t_{1}}^{H_{t_{1}}}-W_{t_{2}}^{H_{t_{2}}}\right|^{p} \leq C_{T, p}\left|t_{1}-t_{2}\right|^{p \min \left(\frac{1}{2}, \beta, h\right)} \forall t_{1}, t_{2} \in[0, T],
$$

where $h=\min _{t \in[0, T]} H_{t}$.

Proof. We refer to [12, page 7].

Theorem 2.1. Assume that $H_{t}$ be a continuously differentiable function.

I. Let $H_{t} \in(0,1)$. RL-mBm $\left\{W_{t}^{H_{t}}, 0 \leq t \leq T\right\}$ can be approximated in $L^{p}(\Omega), p>0$ by semimartingales $W_{t}^{H_{t}, \varepsilon}$ which are defined as follows for every $\varepsilon>0$

$$
W_{t}^{H_{t}, \varepsilon}:=\int_{0}^{t}(t-s+\varepsilon)^{\alpha_{t+\varepsilon}} d W_{s} \quad, \alpha_{t}=H_{t}-\frac{1}{2} .
$$


II. Let $u=\left\{u_{t}, 0 \leq t \leq T\right\}$ be an adapted process bounded in the norm of the space $\mathbb{D}_{W}^{1,2}$. Then

$$
\int_{0}^{t} u_{s} d W_{s}^{H_{s}}=\lim _{\varepsilon \rightarrow 0^{+}} \int_{0}^{t} u_{s} d W_{s}^{H_{s}, \varepsilon} \text { in } L^{2}(\Omega),
$$

uniformly in $t \in[0, T]$, provided that $H_{t}>1 / 2 \forall t \in[0, T]$.

Proof. I. Firstly, we show that $W_{t}^{H_{t}, \varepsilon}$ is a semimartingale. Indeed, we have the following semimartingale decomposition for stochastic Volterra integrals

$$
\int_{0}^{t} g(t, s) d W_{s}=\int_{0}^{t} g(s, s) d W_{s}+\int_{0}^{t}\left(\int_{0}^{s} \frac{\partial}{\partial s} g(s, u) d W_{u}\right) d s,
$$

provided that integrals in the right hand side exist. And then by choosing $g(t, s)=$ $(t-s+\varepsilon)^{\alpha_{t+\varepsilon}}$ we get

$$
W_{t}^{H_{t}, \varepsilon}=\int_{0}^{t} \varepsilon^{\alpha_{s+\varepsilon}} d W_{s}+\int_{0}^{t} \varphi_{s}^{\varepsilon} d s, t \in[0, T],
$$

where $\varphi_{s}^{\varepsilon}=\int_{0}^{s} \partial_{1} K(s+\varepsilon, u) d W_{u}, \partial_{1} K(s+\varepsilon, u)=(s-u+\varepsilon)^{\alpha_{s+\varepsilon}-1}\left[\alpha_{s+\varepsilon}^{\prime}(s-u+\right.$ $\left.\varepsilon) \ln (s-u+\varepsilon)+\alpha_{s+\varepsilon}\right]$.

By the Itô isometry formula and Lemma 2.1 we have

$$
\begin{aligned}
& E\left|W_{t}^{H_{t}, \varepsilon}-W_{t}^{H_{t}}\right|^{2}=E\left|\int_{0}^{t}(t-s+\varepsilon)^{\alpha_{t+\varepsilon}} d W_{s}-\int_{0}^{t}(t-s)^{\alpha_{t}} d W_{s}\right|^{2} \\
= & \int_{0}^{t}(t-s+\varepsilon)^{2 \alpha_{t+\varepsilon}} d s-2 \int_{0}^{t}(t-s+\varepsilon)^{\alpha_{t+\varepsilon}}(t-s)^{\alpha_{t}} d s+\int_{0}^{t}(t-s)^{2 \alpha_{t}} d s \\
\leq & \int_{0}^{t+\varepsilon}(t-s+\varepsilon)^{2 \alpha_{t+\varepsilon}} d s-2 \int_{0}^{t}(t-s+\varepsilon)^{\alpha_{t+\varepsilon}}(t-s)^{\alpha_{t}} d s+\int_{0}^{t}(t-s)^{2 \alpha_{t}} d s \\
= & E\left|W_{t+\varepsilon}^{H_{t+\varepsilon}}-W_{t}^{H_{t}}\right|^{2} \leq C_{T} \varepsilon^{2 \min \left(\frac{1}{2}, \beta, h\right) .}
\end{aligned}
$$

Since both $W_{t}^{H_{t}, \varepsilon}$ and $W_{t}^{H_{t}}$ are Gaussian processes, (2.4) implies that for each $p>0$, there exists $C_{T, p}$ such that

$$
E\left|W_{t}^{H_{t}, \varepsilon}-W_{t}^{H_{t}}\right|^{p} \leq C_{T, p} \varepsilon^{p \min \left(\frac{1}{2}, \beta, h\right)}
$$

which means that $W_{t}^{H_{t}, \varepsilon}$ converges to $W_{t}^{H_{t}}$ in $L^{p}(\Omega), p>0$ when $\varepsilon \rightarrow 0$, uniformly in $t \in[0, T]$. 
II. From the decomposition (2.3) and by the integration by parts formula for the Skorohod integral we have

$$
\begin{aligned}
& \int_{0}^{t} u_{s} d W_{s}^{H, \varepsilon}=\int_{0}^{t} u_{s} \varepsilon^{\alpha_{s+\varepsilon}} d W_{s}+\int_{0}^{t} u_{s} \int_{0}^{s} \partial_{1} K(s+\varepsilon, r) d W_{r} d s \\
= & \int_{0}^{t} u_{s} \varepsilon^{\alpha_{s+\varepsilon}} d W_{s}+\int_{0}^{t} \int_{0}^{s} u_{s} \partial_{1} K(s+\varepsilon, r) \delta W_{r} d s+\int_{0}^{t} \int_{r}^{t} D_{r}^{W} u_{s} \partial_{1} K(s+\varepsilon, r) d s d r \\
= & \int_{0}^{t} u_{s} \varepsilon^{\alpha_{s+\varepsilon}} d W_{s}+\int_{0}^{t} \int_{r}^{t} u_{s} \partial_{1} K(s+\varepsilon, r) d s \delta W_{r}+\int_{0}^{t} \int_{r}^{t} D_{r}^{W} u_{s} \partial_{1} K(s+\varepsilon, r) d s d r .
\end{aligned}
$$

As a consequence,

$$
\begin{aligned}
& E\left|\int_{0}^{t} u_{s} d W_{s}^{H, \varepsilon}-\int_{0}^{t} u_{s} d W_{s}^{H, \varepsilon}\right|^{2} \leq 3 E\left|\int_{0}^{t} u_{s} \varepsilon^{\alpha_{s+\varepsilon}} d W_{s}\right|^{2} \\
+ & 3 E\left|\int_{0}^{t} \int_{r}^{t} u_{s}\left(\partial_{1} K(s+\varepsilon, r)-\partial_{1} K(s, r)\right) d s \delta W_{r}\right|^{2} \\
+ & 3 E\left|\int_{0}^{t} \int_{r}^{t} D_{r}^{W} u_{s}\left(\partial_{1} K(s+\varepsilon, r)-\partial_{1} K(s, r)\right) d s d r\right|^{2}:=3(A+B+C) .
\end{aligned}
$$

It is obvious that $A \rightarrow 0$ as $\varepsilon \rightarrow 0$, uniformly in $t \in[0, T]$. By Meyer's inequality (see, [11]) we have

$$
B \leq \int_{0}^{t}\left\|\int_{r}^{t} u_{s}\left(\partial_{1} K(s+\varepsilon, r)-\partial_{1} K(s, r)\right) d s\right\|_{1,2}^{2} d r
$$

which implies that $B \rightarrow 0$ uniformly in $t \in[0, T]$ as $\varepsilon \rightarrow 0$ because the process $u_{s}$ is bounded in the norm $\|\cdot\|_{1,2}$. Similarly, $C \rightarrow 0$ since

$$
C \leq\left\|\int_{r}^{t} u_{s}\left(\partial_{1} K(s+\varepsilon, r)-\partial_{1} K(s, r)\right) d s\right\|_{1,2}^{2} .
$$

The Theorem thus is proved.

\section{Linear Multifractional Volterra Integro-differential Equation}

In this section, we consider the linear multifractional Volterra integro-differential equation without time delay (1.3). We first prove the existence and uniqueness of the solution with the help of the following lemma. 
Lemma 3.1. Suppose that $G \in L^{1}[0, T]$ and $u \in L^{p}([0, T] \times \Omega), p>1$. Then

$$
E \int_{0}^{t}\left(\int_{0}^{s} G(s-r) u_{r} d r\right)^{p} d s \leq L^{p} \int_{0}^{t} E\left|u_{r}\right|^{p} d r
$$

where $L=\int_{0}^{T} G(s) d s$ being a finite constant.

Proof. An application of Hölder inequality yields

$$
\begin{aligned}
\int_{0}^{t}\left(\int_{0}^{s} G(s-r) u_{r} d r\right)^{p} d s & \leq \int_{0}^{t}\left(\int_{0}^{s} G(s-r) u_{r}^{p} d r\right)\left(\int_{0}^{s} G(s-r) d r\right)^{p-1} d s \\
& \leq L^{p-1} \int_{0}^{t} \int_{r}^{t} G(s-r) u_{r}^{p} d s d r \leq L^{p} \int_{0}^{t} u_{r}^{p} d r
\end{aligned}
$$

The Lemma is proved.

Proposition 3.1. Suppose that $G \in L^{1}[0, T]$ and $\sigma \in \mathbb{D}_{W}^{1,2}\left(\mathcal{H}_{K r}\right)$. Then the equation (1.3) has a unique solution.

Proof. Since the multifractional stochastic term in the right hand side of (1.3) does not contain of $X_{t}$, we can prove our theorem by the classical method of Picard iteration. For all $t \in[0, T]$, consider the functional sequence $\left\{X_{t}^{(n)}, n \geq 0\right\}$ defined by

$$
\left\{\begin{array}{l}
X_{t}^{(0)}=X_{0}+\int_{0}^{t} \sigma_{s} d W_{s}^{H_{s}} \\
X_{t}^{(n+1)}=X_{0}+\int_{0}^{t}\left(a X_{s}^{(n)}+\int_{0}^{s} G(s-u) X_{u}^{(n)} d u\right) d s+\int_{0}^{t} \sigma_{s} d W_{s}^{H_{s}} .
\end{array}\right.
$$

We have

$$
E\left|X_{t}^{(1)}-X_{t}^{(0)}\right|^{2} \leq \int_{0}^{t}\left(a^{2}+L^{2}\right) E\left(\left|X_{0}\right|^{2}+\left|\int_{0}^{s} \sigma_{r} d W_{r}^{H_{r}}\right|^{2}\right) d s:=L_{1}<\infty
$$

uniformly in $t \in[0, T]$. For a generic $\mathrm{n}$, we thus have

$$
\begin{aligned}
& E\left|X_{t}^{(n+1)}-X_{t}^{(n)}\right|^{2} \leq \int_{0}^{t}\left(a^{2}+L^{2}\right) E\left|X_{s}^{(n)}-X_{s}^{(n-1)}\right|^{2} d s \\
& \leq\left(a^{2}+L^{2}\right)^{n-1} \int_{0}^{t} \int_{0}^{s_{1}} \ldots \int_{0}^{s_{n-1}} E\left|X_{s_{n}}^{(1)}-X_{s_{n}}^{(0)}\right|^{2} d s_{n} \ldots d s_{1} \leq \frac{L_{1} T^{n}\left(a^{2}+L^{2}\right)^{n-1}}{n !} .
\end{aligned}
$$


From (3.4) we get the existence and uniqueness of the solution by using standard arguments of the method of iteration.

The first main results of this section are stated in the following.

Theorem 3.1. Suppose that $G \in L^{1}[0, T]$ and $\sigma$ is an adapted process bounded in the norm of the space $\mathbb{D}_{W}^{1,2}$.

I. Consider the linear Volterra integro-differential equation driven by semimartingales

$$
d X_{t}^{\varepsilon}=\left(a X_{t}^{\varepsilon}+\int_{0}^{t} G(t-u) X_{u}^{\varepsilon} d u\right) d t+\sigma_{t} d W_{t}^{H_{t}, \varepsilon}, X_{0}^{\varepsilon}=X_{0}=x .
$$

Then the solution $X_{t}^{\varepsilon}$ of the equation (3.5) converges in $L^{2}(\Omega)$ to the solution $X_{t}$ of the equation (1.3) as $\varepsilon$ tends to zero.

II. The unique solution $X_{t}$ of the equation (1.3) admits the representation which is so-called the variation of parameters formula

$$
X_{t}=Z_{t} X_{0}+\int_{0}^{t} Z_{t-s} \sigma_{s} d W_{s}^{H_{s}}
$$

where $Z_{t}$ is a unique solution of the resolvent equation

$$
d Z_{t}=\left(a Z_{t}+\int_{0}^{t} G(t-u) Z_{u} d u\right) d t, Z_{0}=1
$$

Proof. I. We have

$$
\begin{gathered}
X_{t}=X_{0}+\int_{0}^{t}\left(a X_{s}+\int_{0}^{s} G(s-u) X_{u} d u\right) d s+\int_{0}^{t} \sigma_{s} d W_{s}^{H_{s}}, \\
X_{t}^{\varepsilon}=X_{0}+\int_{0}^{t}\left(a X_{s}^{\varepsilon}+\int_{0}^{s} G(s-u) X_{u}^{\varepsilon} d u\right) d s+\int_{0}^{t} \sigma_{s} d W_{s}^{H_{s}, \varepsilon} .
\end{gathered}
$$

Hence,

$$
\begin{aligned}
E\left|X_{t}^{\varepsilon}-X_{t}\right|^{2} \leq & 3 a^{2} \int_{0}^{t} E\left|X_{s}^{\varepsilon}-X_{s}\right|^{2} d s+3 E \int_{0}^{t}\left(\int_{0}^{s} G(s-u)\left|X_{u}^{\varepsilon}-X_{u}\right| d u\right)^{2} d s \\
& +3 E\left|\int_{0}^{t} \sigma_{s} d\left(W_{s}^{H_{s}, \varepsilon}-W_{s}^{H_{s}}\right)\right|^{2}
\end{aligned}
$$


By Lemma 3.1 we get

$$
E\left|X_{t}^{\varepsilon}-X_{t}\right|^{2} \leq 3\left(a^{2}+L^{2}\right) \int_{0}^{t} E\left|X_{s}^{\varepsilon}-X_{s}\right|^{2} d s+f_{\varepsilon}(t),
$$

where $f_{\varepsilon}(t)=3 E\left|\int_{0}^{t} \sigma_{s} d\left(W_{s}^{H_{s}, \varepsilon}-W_{s}^{H_{s}}\right)\right|^{2}$ that converges to zero as $\varepsilon \rightarrow 0$, uniformly in $t \in[0, T]$.

Applying a generalization of the Gronwall-Bellman inequality (see, [6]) to (3.8) we get

$$
E\left|X_{t}^{\varepsilon}-X_{t}\right|^{2} \leq f_{\varepsilon}(t)+4\left(a^{2}+L^{2}\right) \int_{0}^{t} f_{\varepsilon}(s) e^{4\left(a^{2}+L^{2}\right)(t-s)} d s .
$$

We finish the proof by dominated convergence theorem.

II. Firstly, we consider the following two equations with the initial conditions $U_{0}, V_{0}$ such that $U_{0}+V_{0}=X_{0}$

$$
\begin{gathered}
d U_{t}^{\varepsilon}=\left(a U_{t}^{\varepsilon}+\int_{0}^{t} G(t-u) U_{u}^{\varepsilon} d u\right) d t+\varepsilon^{\alpha_{t+\varepsilon}} \sigma_{t} d W_{t}, \\
d V_{t}^{\varepsilon}=\left(a V_{t}^{\varepsilon}+\int_{0}^{t} G(t-u) V_{u}^{\varepsilon} d u+\sigma_{t} \varphi_{t}^{\varepsilon}\right) d t .
\end{gathered}
$$

The equation (3.10) is a stochastic Volterra integro-differential equation in the sense of Itô and for every fixed $\omega \in \Omega,(3.11)$ is a deterministic Volterra integro-differential equation. And then by the classical variation of parameters formula we have

$$
\begin{gathered}
U_{t}^{\varepsilon}=Z_{t} U_{0}+\int_{0}^{t} Z_{t-s} \varepsilon^{\alpha_{s+\varepsilon}} \sigma_{s} d W_{s}, \\
V_{t}^{\varepsilon}=Z_{t} V_{0}+\int_{0}^{t} Z_{t-s} \varphi_{s}^{\varepsilon} \sigma_{s} d s .
\end{gathered}
$$

On the other hand, from the decomposition (2.3) we see that $X_{t}^{\varepsilon}=U_{t}^{\varepsilon}+V_{t}^{\varepsilon}$ is solution of (3.5). Consequently,

$$
X_{t}^{\varepsilon}=Z_{t} X_{0}+\int_{0}^{t} Z_{t-s} \sigma_{s} d W_{s}^{H_{s}, \varepsilon} .
$$

The formula (3.6) follows directly from (3.12) by taking the limit in $L^{2}(\Omega)$ as $\varepsilon \rightarrow 0$.

The Theorem thus is proved. 
Corollary 3.1. The solution of multifractional Langevin equation

$$
d X_{t}=-b X_{t} d t+\sigma d W_{t}^{H_{t}}
$$

is unique and given by

$$
X_{t}=e^{-b t} X_{0}+\int_{0}^{t} \sigma e^{-b(t-s)} d W_{s}^{H_{s}} .
$$

We now give a sufficient condition for the almost sure exponential convergence of the solution. Thank to the variation of parameters formula (3.6), this can be done via exponentially asymptotic stability given by Murakami [10] for the deterministic Volterra equations:

$$
d x_{t}=\left(a x_{t}+\int_{0}^{t} G(t-u) x_{u} d u\right) d t, t \geq t_{0}
$$

the initial condition $x_{t}=\phi(t), t \in\left[0, t_{0}\right]$, where $\phi \in C\left(\left[0, t_{0}\right], \mathbb{R}\right)$.

Lemma 3.2. Suppose that $f \in C\left(\mathbb{R}^{+}, \mathbb{R}^{+}\right) \cap L^{1}\left(\mathbb{R}^{+}\right)$satisfies

$$
\int_{0}^{\infty} f(t) e^{\gamma t} d t<\infty \text { for some } \gamma>0 .
$$

If $\lambda>0$ and $\lambda^{\prime}=\lambda \wedge \gamma$ then

$$
\int_{0}^{t} e^{-\lambda(t-s)} f(s) d s<e^{-\lambda^{\prime} t} \int_{0}^{\infty} f(s) e^{\gamma s} d s .
$$

Proof. Refer to [2, Lemma 3.1].

We now are position to formulate the next main results of this section.

Theorem 3.2. Suppose that $\sigma$ is a deterministic function and that the zero solution of (3.13) is uniformly asymptotically stable, and that the following conditions hold

$$
\int_{0}^{\infty}|G(s)| e^{\lambda_{1} s} d s<\infty \text { for some } \lambda_{1}>0
$$

$$
\int_{0}^{\infty} \sigma_{s}^{2}\left(\frac{\alpha_{s}^{\prime}}{\alpha_{s}+1} s^{\alpha_{s}+1}\left(\ln s-\frac{1}{\alpha_{s}+1}\right)+s^{\alpha_{s}}\right) e^{2 \lambda_{2} s} d s<\infty \text { for some } \lambda_{2}>0
$$


Then

I. there exists $\gamma>0$ and $M_{p}\left(X_{0}\right)>0$ such that for each $p>0$, the solution of (1.3) satisfies

$$
E\left|X_{t}\right|^{p} \leq M_{p}\left(X_{0}\right) e^{-p \gamma t}, t \geq 0 .
$$

II. the solution of (1.3) is almost surely exponentially convergent, i.e. there exists $\beta_{0}>0$ such that

$$
\limsup _{t \rightarrow \infty} \frac{1}{t} \log \left|X_{t}\right| \leq-\beta_{0}, \text { a.s. }
$$

Proof. I. We observe that if $u$ is a deterministic function then

$$
\int_{0}^{t} u_{s} d W_{s}^{H_{s}}=\int_{0}^{t}\left(\int_{r}^{t} u_{s} \partial_{1} K(s, r) d s\right) d W_{r},
$$

where $d W_{r}$ is the classical Ito differential. Thus, for each $t$ the integral $\int_{0}^{t} u_{s} d W_{s}^{H_{s}}$ is centered Gaussian random variable with the variance satisfying the following estimate

$$
\begin{aligned}
& E\left(\int_{0}^{t} u_{s} d W_{s}^{H_{s}}\right)^{2}=\int_{0}^{t}\left(\int_{r}^{t} u_{s} \partial_{1} K(s, r) d s\right)^{2} d r \\
\leq & \int_{0}^{t}\left(\int_{r}^{t}\left|u_{s}\right|^{2} \partial_{1} K(s, r) d s\right) K(t, r) d r \\
\leq & t^{\alpha_{t}} \int_{0}^{t} \int_{r}^{t}\left|u_{s}\right|^{2} \partial_{1} K(s, r) d s d r=t^{\alpha_{t}} \int_{0}^{t} \int_{0}^{s}\left|u_{s}\right|^{2} \partial_{1} K(s, r) d r d s \\
= & t^{\alpha_{t}} \int_{0}^{t}\left|u_{s}\right|^{2}\left(\frac{\alpha_{s}^{\prime}}{\alpha_{s}+1} s^{\alpha_{s}+1}\left(\ln s-\frac{1}{\alpha_{s}+1}\right)+s^{\alpha_{s}}\right) d s .
\end{aligned}
$$

Let $Z_{t}$ be the solution of (3.7). Since the zero solution of (3.13) is uniformly asymptotically stable and (3.14) holds, there exists $C>0$ and $\lambda>0$ such that (see, $[10,2])$

$$
\left|Z_{t}\right| \leq C e^{-\lambda t}, \forall t \geq 0 .
$$

From (3.6) and using the estimates (3.18), (3.19) we have

$$
\begin{aligned}
& E\left|X_{t}-Z_{t} X_{0}\right|^{2} \\
\leq & t^{\alpha_{t}} \int_{0}^{t}\left|Z_{t-s} \sigma_{s}\right|^{2}\left(\frac{\alpha_{s}^{\prime}}{\alpha_{s}+1} s^{\alpha_{s}+1}\left(\ln s-\frac{1}{\alpha_{s}+1}\right)+s^{\alpha_{s}}\right) d s \\
\leq & C^{2} t^{\alpha_{t}} \int_{0}^{t} e^{-2 \lambda(t-s)}\left|\sigma_{s}\right|^{2}\left(\frac{\alpha_{s}^{\prime}}{\alpha_{s}+1} s^{\alpha_{s}+1}\left(\ln s-\frac{1}{\alpha_{s}+1}\right)+s^{\alpha_{s}}\right) d s .
\end{aligned}
$$


Let $\lambda^{\prime}=\min \left(\lambda, \lambda_{2}\right)$ and $\gamma=\frac{\lambda^{\prime}}{2}$. By Lemma 3.2

$$
\begin{aligned}
& E\left|X_{t}-Z_{t} X_{0}\right|^{2} \\
\leq & C^{2} t^{\alpha_{t}} e^{-2 \lambda^{\prime} t} \int_{0}^{\infty}\left|\sigma_{s}\right|^{2}\left(\frac{\alpha_{s}^{\prime}}{\alpha_{s}+1} s^{\alpha_{s}+1}\left(\ln s-\frac{1}{\alpha_{s}+1}\right)+s^{\alpha_{s}}\right) e^{2 \lambda_{2} s} d s \\
\leq & C_{2}^{2} e^{-2 \gamma t},
\end{aligned}
$$

where $C_{2}^{2}=C_{1} \int_{0}^{\infty}\left|\sigma_{s}\right|^{2}\left(\frac{\alpha_{s}^{\prime}}{\alpha_{s}+1} s^{\alpha_{s}+1}\left(\ln s-\frac{1}{\alpha_{s}+1}\right)+s^{\alpha_{s}}\right) e^{2 \lambda_{2} s} d s$ and $C_{1}=\sup _{t \in(0, \infty)}$ $C^{2} t^{\alpha_{t}} e^{-\lambda^{\prime} t}$

In order to establish (3.16) we need two technical results:

(i) Let $Y$ denote a random variable following an $N\left(0, \sigma^{2}\right)$ law. Then for any $p>0$ we have

$$
E|Y|^{p}=\frac{2^{p / 2} \Gamma\left(\frac{p+1}{2}\right)}{\Gamma\left(\frac{1}{2}\right)} \sigma^{p} .
$$

(ii) $(a+b)^{p} \leq c_{p}\left(a^{p}+b^{p}\right) \forall a, b>0$, where $c_{p}=1$ if $0<p \leq 1$ and $c_{p}=2^{p-1}$ if $p>1$.

We now have

$$
\begin{aligned}
E\left|X_{t}\right|^{p} & \leq c_{p}\left(E\left|X_{t}-Z_{t} X_{0}\right|^{p}+\left|Z_{t} X_{0}\right|^{p}\right) \\
& =c_{p}\left(\frac{2^{p / 2} \Gamma\left(\frac{p+1}{2}\right)}{\Gamma\left(\frac{1}{2}\right)}\left(E\left|X_{t}-Z_{t} X_{0}\right|^{2}\right)^{p / 2}+\left|Z_{t} X_{0}\right|^{p}\right) \\
& =c_{p}\left(\frac{2^{p / 2} \Gamma\left(\frac{p+1}{2}\right)}{\Gamma\left(\frac{1}{2}\right)} C_{2}^{p} e^{-p \gamma t}+\left|X_{0}\right|^{p} C^{p} e^{-p \lambda t}\right) \\
& \leq M_{p}\left(X_{0}\right) e^{-p \gamma t}
\end{aligned}
$$

where $M_{p}\left(X_{0}\right)=c_{p}\left(\frac{2^{p / 2} \Gamma\left(\frac{p+1}{2}\right)}{\Gamma\left(\frac{1}{2}\right)} C_{2}^{p}+\left|X_{0}\right|^{p} C^{p}\right)$.

II. We use the Theorem 4.3.1 in [9] to prove (3.17), it is enough to show that there exists a constant $\gamma_{1}>0$ such that

$$
E\left(\sup _{n-1 \leq t<n}\left|X_{t}\right|^{2}\right) \leq M e^{-\gamma_{1}(n-1)} \forall n \geq 1
$$

Indeed, if (3.22) is true then $\beta_{0}=\frac{\gamma_{1}}{2}$.

We have for each $t \geq n-1$

$$
X_{t}=X_{n-1}+\int_{n-1}^{t}\left(a X_{s}+\int_{0}^{s} G(s-u) X_{u} d u\right) d s+\int_{n-1}^{t} \sigma_{s} d W_{s}^{H_{s}}
$$


and then

$$
\begin{aligned}
& E\left(\sup _{n-1 \leq t \leq n}\left|X_{t}\right|^{2}\right) \\
& \leq 3\left(E\left|X_{n-1}\right|^{2}+E \int_{n-1}^{n}\left(a X_{s}+\int_{0}^{s} G(s-u) X_{u} d u\right)^{2} d s\right. \\
& \left.\quad+E\left(\sup _{n-1 \leq t \leq n}\left|\int_{n-1}^{t} \sigma_{s} d W_{s}^{H_{s}}\right|^{2}\right)\right) \\
& \leq 3\left(E\left|X_{n-1}\right|^{2}+\left(a^{2}+L^{2}\right) \int_{n-1}^{n} E\left|X_{s}\right|^{2} d s\right. \\
& \left.\quad+E\left(\sup _{n-1 \leq t \leq n}\left|\int_{n-1}^{t} \sigma_{s} d W_{s}^{H_{s}}\right|^{2}\right)\right) .
\end{aligned}
$$

By the Burkholder-Davis-Gundy inequality, there exists $C_{3}>0$ such that

$$
\begin{aligned}
& E\left(\sup _{n-1 \leq t \leq n}\left|\int_{n-1}^{t} \sigma_{s} d W_{s}^{H_{s}}\right|^{2}\right) \leq C_{3} \int_{n-1}^{n}\left(\int_{r}^{n} \sigma_{s} \partial_{1} K(s, r) d s\right)^{2} d r \\
\leq & C_{3} \int_{n-1}^{n}\left|\sigma_{s}\right|^{2}\left(\frac{\alpha_{s}^{\prime}}{\alpha_{s}+1} s^{\alpha_{s}+1}\left(\ln s-\frac{1}{\alpha_{s}+1}\right)+s^{\alpha_{s}}\right) d s \\
\leq & C_{3} e^{-2 \lambda_{2}(n-1)} \int_{n-1}^{\infty}\left|\sigma_{s}\right|^{2}\left(\frac{\alpha_{s}^{\prime}}{\alpha_{s}+1} s^{\alpha_{s}+1}\left(\ln s-\frac{1}{\alpha_{s}+1}\right)+s^{\alpha_{s}}\right) e^{2 \lambda_{2} s} d s \\
\leq & C_{4} e^{-2 \lambda_{2}(n-1)},
\end{aligned}
$$

where $C_{4}=C_{3} \int_{0}^{\infty}\left|\sigma_{s}\right|^{2}\left(\frac{\alpha_{s}^{\prime}}{\alpha_{s}+1} s^{\alpha_{s}+1}\left(\ln s-\frac{1}{\alpha_{s}+1}\right)+s^{\alpha_{s}}\right) e^{2 \lambda_{2} s} d s<\infty$.

Combining (3.16), (3.23) and (3.24) we get

$$
\begin{aligned}
E\left(\sup _{n-1 \leq t<n}\left|X_{t}\right|^{2}\right) & \leq 3\left(\left(M_{2}\left(X_{0}\right)+a^{2}+L^{2}\right) e^{-2 \gamma(n-1)}+C_{4} e^{-2 \lambda_{2}(n-1)}\right) \\
& \leq M e^{-\gamma_{1}(n-1)}
\end{aligned}
$$

where $\gamma_{1}=2 \gamma$ and $M=M_{2}\left(X_{0}\right)+a^{2}+L^{2}+C_{4}$.

The Theorem thus is proved.

Remark 3.1. In the classical case, where RL-mBm reduces to a standard Brownian motion, the exponential convergence of the solution is discussed more details by Appleby and Alan in [2]. 


\section{Multifractional Volterra Integro-differential Equation with Time Delay}

The aim of the section is to prove the existence and uniqueness of the solution of the multifractional stochastic Volterra integro-differential equation with time delay:

$$
d X_{t}=\left(a X_{t}+\int_{0}^{t} G(t-u) X_{u} d u\right) d t+b\left(X_{t-r}\right) d W_{t}^{H_{t}}, t \in[0, T],
$$

$X_{t}=\phi(t), t \in[-r, 0]$, where $\phi \in C[-r, 0]$.

Noting that since multifractional stochastic integral defined in Definition 2.1 contains of Skorohod integral and Malliavin derivative, we cannot use traditional methods to prove the existence and uniqueness of the solution. However, fortunately, the delay time, $r$, is discrete we can use the method of induction to do this. Our idea is as follows: first prove the result for the interval $[0, r]$, then we use this solution process as the initial condition to solve the equation within the interval $[r, 2 r]$, and so on. We need the following two lemmas.

Lemma 4.1. Let $u$ be a adapted stochastic process bounded in the norm of the space $\mathbb{D}_{W}^{1,2}$ such that for all $r \in[0, T]$, stochastic process $D_{r}^{W} u$ also does. We have for any $r \leq t$

$$
D_{r}^{W}\left(\int_{0}^{t} u_{s} d W_{s}^{H_{s}}\right)=\int_{r}^{t} D_{r}^{W} u_{s} d W_{s}^{H_{s}}+\int_{r}^{t} u_{s} \partial_{1} K(s, r) d s .
$$

Proof. From the decomposition (2.3) we have

$$
\int_{0}^{t} u_{s} d W_{s}^{H, \varepsilon}=\int_{0}^{t} \varepsilon^{\alpha_{s+\varepsilon}} u_{s} d W_{s}+\int_{0}^{t} u_{s} \varphi_{s}^{\varepsilon} d s .
$$

By taking Malliavin derivative

$$
\begin{aligned}
& D_{r}^{W}\left(\int_{0}^{t} u_{s} d W_{s}^{H, \varepsilon}\right) \\
& =\varepsilon^{\alpha_{r+\varepsilon}} u_{r}+\int_{r}^{t} \varepsilon^{\alpha_{s+\varepsilon}} D_{r}^{W} u_{s} d W_{s}+\int_{r}^{t} \varphi_{s}^{\varepsilon} D_{r}^{W} u_{s} d s+\int_{r}^{t} u_{s} \partial_{1} K(s+\varepsilon, r) d s \\
& =\varepsilon^{\alpha_{r+\varepsilon}} u_{r}+\int_{r}^{t} D_{r}^{W} u_{s} d W_{s}^{H_{s}, \varepsilon}+\int_{r}^{u_{s} \partial_{1} K(s+\varepsilon, r) d s}
\end{aligned}
$$

Taking limit above expression in $L^{2}(\Omega)$ as $\varepsilon \rightarrow 0$ and by closability of Malliavin derivative we get desired result. 
For fixed $k \geq 1$, we denote by $\mathfrak{D}^{k, 2}$ the space of stochastic processes such that $u \in \mathbb{D}_{W}^{k, 2}$ and

$$
\sup _{s \in[0, T]} E\left|u_{s}\right|^{2}+\sup _{r_{1}, \ldots, r_{l}, s \in[0, T]} E\left|D_{r_{1}, \ldots, r_{l}}^{W, l} u_{s}\right|^{2} \leq L_{l}
$$

for any $l \leq k$, where $L_{l}$ is a finite constant.

By Proposition 2.1 we have $\mathfrak{D}^{k, 2} \subset \mathbb{D}_{W}^{1,2}\left(\mathcal{H}_{K}\right)$.

Lemma 4.2. Suppose that $\sigma \in \mathfrak{D}^{k+1,2}$. The unique solution $X_{t}$ of (1.3) belongs to $\mathfrak{D}^{k, 2}$.

Proof. Let $Z$ is the solution of the resolvent equation (3.7). Since $Z$ is a continuous deterministic function and $\sigma \in \mathfrak{D}^{k+1,2}$, we have $\sigma_{s} Z_{t-s}$ belongs to $\mathfrak{D}^{k+1,2}$. From Lemma 4.1 and by using the method of induction, $\left\{\int_{0}^{t} \sigma_{s} Z_{t-s} d W_{s}^{H_{s}}, t \in[0, T]\right\} \in$ $\mathfrak{D}^{k, 2}$. Consequently, the fact the solution $X_{t}$ of (1.3) belongs to $\mathfrak{D}^{k, 2}$ follows from the variation of parameters formula (3.6).

Theorem 4.1. Suppose that $b(x)$ is $N$-order differentiable function with bounded derivatives, where $N$ is the smallest integer not smaller than $\frac{T}{r}$ and that $b(0)$ is bounded. Then (4.1) admits a unique solution in $[0, T]$. Moreover, the solution can be explicitly expressed by

$$
\begin{cases}X_{t}=\phi(t) & t \in[-r, 0] \\ X_{t}=Z_{t} X_{0}+\int_{0}^{t} Z_{t-s} b\left(X_{s-r}\right) d W_{s}^{H_{s}}, & t \geq 0\end{cases}
$$

where $Z_{t}$ is the solution of the resolvent equation.

Proof. For simplicity let us assume $T=N r$, where $N$ is a positive integer number. Our induction hypothesis, for $n<N$, is the following:

$\left(H_{n}\right)$ The equation

$$
X_{t}=\phi(0)+\int_{0}^{t}\left(a X_{s}+\int_{0}^{s} G(s-u) X_{u} d u\right) d s+\int_{0}^{t} b\left(X_{s-r}\right) d W_{s}^{H_{s}}, t \in[0, n r]
$$

with $X_{t}=0, t>n r$, has a unique solution in $\mathfrak{D}^{N-n, 2}$.

We check $\left(H_{1}\right)$ at first. Let $t \in[0, r]$, the equation (4.4) now becomes

$$
X_{t}=\phi(0)+\int_{0}^{t}\left(a X_{s}+\int_{0}^{s} G(s-u) X_{u} d u\right) d s+\int_{0}^{t} b(\phi(s-r)) d W_{s}^{H_{s}} \quad t \in[0, r]
$$

It is obvious that $\sigma_{s}:=b(\phi(s-r)) \in \mathfrak{D}^{N, 2}$ because $\phi$ is a continuous deterministic function. From Lemma 4.2 we have that (4.5) has a unique solution and that this solution belongs to $\mathfrak{D}^{N-1,2}$. Thus, $\left(H_{1}\right)$ is true. 
We now assume that $\left(H_{i}\right)$ is true for any $i \leq n$, where $n<N$. We need to check that $\left(H_{n+1}\right)$ is also true. Put

$$
Y_{t}= \begin{cases}\phi(t-r), & 0 \leq t \leq r \\ X_{t-r}, & r<t \leq(n+1) r \\ 0, & t>(n+1) r\end{cases}
$$

where $X$ is the solution found in $\left(H_{n}\right)$. It is easy to see that $Y \in \mathfrak{D}^{N-n, 2}$ since $X \in \mathfrak{D}^{N-n, 2}$ and

$$
D_{s}^{W, k} Y_{t}= \begin{cases}D_{s}^{W, k} X_{t-r}, & r<t \leq(n+1) r \\ 0, & \text { otherwise. }\end{cases}
$$

The equation (4.4) now becomes (1.3):

$$
X_{t}=\phi(0)+\int_{0}^{t}\left(a X_{s}+\int_{0}^{s} G(s-u) X_{u} d u\right) d s+\int_{0}^{t} \sigma_{s} d W_{s}^{H_{s}}, t \in[0,(n+1) r]
$$

where $\sigma_{s}=b\left(Y_{s}\right)$.

Since $b(x)$ has bounded derivatives up to order $N, b(0)$ is bounded and $Y \in$ $\mathfrak{D}^{N-n, 2}$, it is clear that $\sigma \in \mathfrak{D}^{N-n, 2}$. As a consequence, (4.6) has a unique solution and this solution belongs to $\mathfrak{D}^{N-n-1,2}$.

We complete the proof of this theorem.

\section{ACKNOWLEDGMENT}

The author would like to thank the anonymous referees for their valuable comments for improving the paper.

This research is funded by Vietnam National Foundation for Science and Technology Development (NAFOSTED) under grant number 101.02-2011.12 (16-101).

\section{REFERENCES}

1. E. Alòs, O. Mazet and D. Nualart, Stochastic Calculus with Respect to Gaussian Processes, The Annals of Probability, 29(2) (2001), 766-801.

2. J. A. D. Appleby and A. Freeman, Exponential asymptotic stability of linear Itô-Volterra equations with damped stochastic perturbations, Electron. J. Probab., 8(22) (2003), 22.

3. P. R. Bertrand, A. Hamdouni and S. Khadhraoui, Modelling NASDAQ Series by Sparse Multifractional Brownian Motion, Methodology and Computing in Applied Probability, (23 July 2010), pp. 1-18.

4. T. A. Burton, Volterra integral and differential equations, Mathematics in Science and Engineering, 2nd ed., 202, Elsevier B. V., Amsterdam, 2005. 
5. B. Boufoussi, M. Dozzi and R. Marty, Local time and Tanaka formula for a Volterra-type multifractional Gaussian process, Bernoulli, 16(4) (2010), 1294-1311.

6. H. E. Gollwitzer, A note on a functional inequality, Proc. Amer. Math. Soc., 23 (1969), 642-647.

7. M. Li, S. C. Lim, B. J. Hu and H. Feng, Towards describing multi-fractality of traffic using local Hurst function, in: Lecture Notes in Computer Science, Vol. 4488, Springer, 2007, pp. 1012-1020.

8. S. C. Lim, Fractional Brownian motion and multifractional Brownian motion of RiemannLiouville type, J. Phys. A: Math. Gen., 34 (2001), 1301-1310.

9. X. Mao, Exponential Stability of Stochastic Differential Equations, Vol. 182 of Pure and Applied Mathematics, Marcel Dekker, New York, 1994.

10. S. Murakami, Exponential asymptotic stability for scalar linear Volterra equations, Differential Integral Equations, 4(2) (1991), 519-525.

11. D. Nualart, The Malliavin Calculus and Related Topics, 2nd ed., Springer, 2006.

12. R. F. Peltier and J. Lévy-Véhel, Multifractional brownian motion: definition and preliminary results, Rapport de recherche de l'INRIA, 2645, 1995.

13. N. Privault, Skorohod stochastic integration with respect to non-adapted processes on Wiener space, Stochastics Stochastics Rep., 65(1-2) (1998), 13-39.

\author{
Nguyen Tien Dung \\ Department of Mathematics \\ FPT University \\ No 8 Ton That Thuyet \\ Cau Giay, Hanoi \\ VietNam \\ E-mail: dung_nguyentien10@yahoo.com \\ dungnt@fpt.edu.vn
}

